\title{
The Application of Contours Comparison Methods for Matching Segmented Point Clouds
}

\author{
Andrei Nikitin ${ }^{1, *}$, Timur Mazitov ${ }^{1}$ \\ ${ }^{1}$ Izhevsk State Technical University, ul. Studencheskaya 7, Izhevsk, 426069 Russia
}

\begin{abstract}
This paper is devoted to the application of methods for contours and shapes comparison of objects represented as two-dimensional point clouds obtained during segmentation of data from a laser scanning system. The methods considered allow estimating the displacement of objects in the field of view of the scanning system. Methods are considered on the basis of Euclidean distances, Hausdorff metrics, invariant moments and shape context.
\end{abstract}

Keywords: shape; contour; contour comparison; Euclidean distance; invariant moments; Hausdorff metric; shape context; technical vision; image processing.

\section{Introduction}

Data segmentation of scanning systems, objects segmentation, matching and tracking are complex tasks in perspective and developing areas of technical vision and image processing. The methods of comparing segmented data volumes, twodimensional or three-dimensional point clouds, whole parameters of objects or images, allow to estimate changes in the data, reduce the amount of processed information, and use real-time data processing systems.

Processing visual data of scanning systems makes it possible to estimate contours for observable objects their shape is determined. Contours and shapes of objects can be used to determine changes in the object over time, in particular to determine the movement's vector in the reference frame relative to the scanning system, i.e. to accomplish the task of object tracking, or determine whether an object is moving or not.

To compare contours, a large number of algorithms and techniques are proposed that work with different representations of contours - a set of two-dimensional points, or a binary image. In [1] invariant moments are used to recognize symmetric objects. The authors of [2] apply the Hausdorff metric for searching text on binary images. In [3] is used the method of pattern recognition by the context of forms. In [4] is presented a modification of the Hausdorff metric and its application in Euclidean space. In [5], for contours comparison, along with invariant moments, the morphing method and methods based on Fourier descriptors and polar diagrams are applied.

In this paper we use segmented two-dimensional point clouds obtained from the laser scanning system and transformed to a single reference point using spatial matching methods, since some of the using methods are non-invariant to rotation. For segments, the spatial coordinates of the points are defined, combined into contours for comparison. 


\section{Methods overview}

\subsection{Euclidean metric}

The first comparison method is based on the Euclidean metric [6], one of the most common methods for determining the distance between points. The distance between two points is determined by the formula:

$$
\rho(A, B)=\sqrt{\left(x_{2}-x_{1}\right)^{2}+\left(y_{2}-y_{1}\right)^{2}}
$$

Since the number of points in contours of the segments is different, it is necessary to solve the problem of finding the nearest points, and then to normalize the obtained metric. For that searching through the k-dimensional tree is used.

\subsection{Hausdorff metric}

The second comparison method is based on the Hausdorff metric [6] [7]. The Hausdorff metric is calculated in the space of curves, which are the contours of the segments. If we denote as $x$ the points of the contour $u$ of the first segment, $y$ as the points of the contour $w$ of the second segment, then the radius of the points in relation to the contours is equal to:

$$
\begin{gathered}
R_{u}=\max _{\substack{y \in w \\
x \in u}} \min \rho(x, y) \\
R_{w}=\max _{\substack{x \in \mathcal{u} \\
y \in w}} \min \rho(x, y)
\end{gathered}
$$

On this basis, the Hausdorff metric $\rho_{H}$ equals:

$$
\rho_{H}(u, w)=\max \left(R_{u}, R_{w}\right)
$$

\subsection{Invariant moments}

The method of invariant moments is used in the area of image processing and analysis [8,9] and is a particular weighted average function of the pixel intensities of the image. Image moments have long been used to describe objects after their segmentation and determine the properties of the area, geometric center and orientation. This method used in this paper for the two-dimensional vector of points, transformed into a binary image.

The initial moment for the two-dimensional function $f(x, y)$ of degree $p, q(1,2 \ldots)$ for the area $\mathrm{D}$ is equal to:

$$
M_{p q}=\iint_{D} x^{p} y^{q} f(x, y) d x d y
$$

The central moment $\mu_{p q}$, providing the invariance to the shift, is equal to:

$$
\begin{aligned}
& \mu_{p q}=\iint_{D}(x-\bar{x})^{p}(y-\bar{y})^{q} f(x, y) d x d y \\
& \bar{x}=\frac{M_{10}}{M_{00}}, \quad \bar{y}=\frac{M_{01}}{M_{00}}
\end{aligned}
$$

Where, $\bar{x}$ and $\bar{y}$ are the coordinates of the center of the area $\mathrm{D}$. The transition to normalized central moments provides invariance to scaling:

$$
\eta_{p q}=\frac{\mu_{p q}}{\mu_{0 \mathbf{0}}\left(\frac{p+q}{2}+1\right)}
$$

The method also provides rotation invariance [8].

\subsection{Shape Context}

The shape context method is used in the pattern recognition and proposed in [3]. This characteristic is intended to describe the shapes in order to measure their similarity and restore point correspondences. The basic idea is to choose $n$ points of the shape contour. For each point pi of the form $n-1$ vectors obtained by connecting the point $p$ with all other points are considered. The set of these vectors is called the description of the shape localized at this point. The main idea is the distribution of the point relative coordinates is its reliable and compact identifier.

For point $p_{i}$, the histogram $h_{i}$ of the relative coordinates for the remaining $n-1$ points is:

$$
h_{i}(k)=\#\left\{q-p_{i}:\left(q-p_{i}\right) \in \operatorname{bin}(k)\right\}
$$

This histogram $h_{i}$ is the context of the form for the point $p_{i}$, bin is the column of the histogram in polar coordinates, $q$ is the point for comparison.

\section{Experimental results}

For contours comparison were obtained sets of room scans from the laser scanning system with one and two moving objects.

To prevent wrong contours matching were introduced threshold values for the similarity measure of contours and the distance between matched contours. 


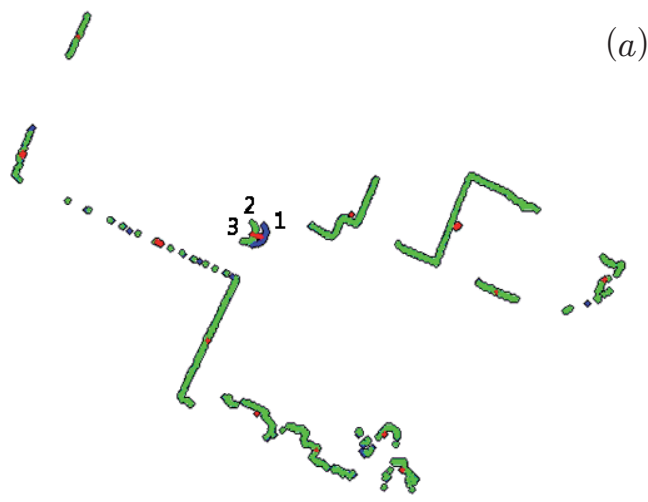

I

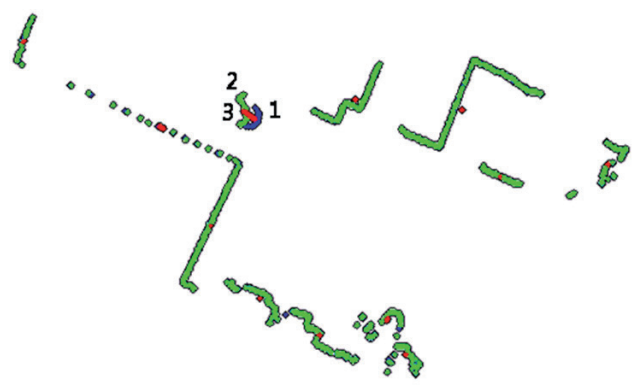

Fig. 1: An example of the comparison algorithm result by the method of Euclidean distances with one moving object. (a) Previous scan. (b) Current scan. On figure: 1 is the previous position of the moving object, 2 is current position of the moving object, $\mathbf{3}$ is the positions connecting line.

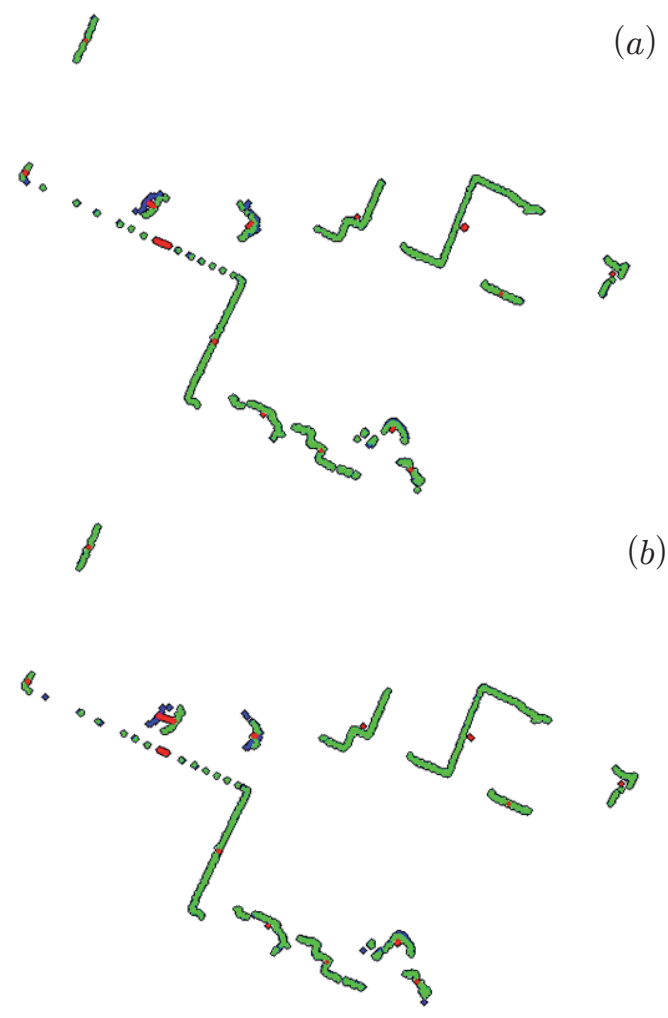

Fig. 2: An example of the comparison algorithm result by the method of Euclidean distances with two moving objects. (a) Previous scan. (b) Current scan.

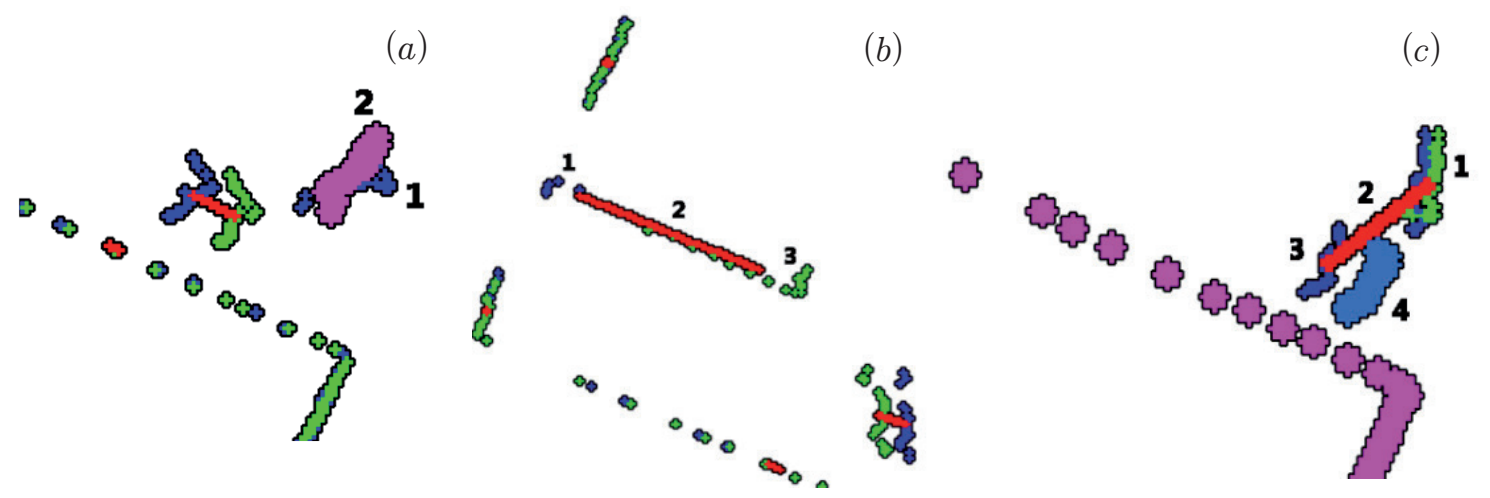

Fig. 3: An example of errors by contours matching. (a) No specific contour for the first dynamic object. On this part of figure: 1 is previous position of first moving object, 2 is current unmatched position of first moving object. (b) The determination of similar contours at a large distance when the true contour disappears from the field of view of scanning system. On this part of figure: 1 is the previous position of the object, 2 is the positions connecting line, 3 is current position of the object. (c) Wrong moving object matching. On this part of figure: 1 is the current position of the first moving object, 2 is the positions connecting line, 3 is previous position of the second moving object, 4 unmatched current position of second moving object. 
Tab. 1: The results of the operation of comparison methods with the similarity measure threshold value for environment with one moving object.

\begin{tabular}{|l|l|l|l|l|}
\hline & $\begin{array}{l}\text { Euclidean } \\
\text { distance }\end{array}$ & $\begin{array}{l}\text { Hausdorff } \\
\text { metric }\end{array}$ & $\begin{array}{l}\text { Invariant } \\
\text { image } \\
\text { moments }\end{array}$ & $\begin{array}{l}\text { Shape } \\
\text { context }\end{array}$ \\
\hline Wrong matching (\%) & 1.1 & 0.9 & 8.2 & 9.2 \\
\hline $\begin{array}{l}\text { No matched contours } \\
(\%)\end{array}$ & 1.7 & 1.5 & 17.5 & 3.1 \\
\hline
\end{tabular}

Tab. 2: The results of the operation of comparison methods with the similarity measure threshold value for environment with two moving objects.

\begin{tabular}{|l|l|l|l|l|}
\hline & $\begin{array}{l}\text { Euclidean } \\
\text { distance }\end{array}$ & $\begin{array}{l}\text { Hausdorff } \\
\text { metric }\end{array}$ & $\begin{array}{l}\text { Invariant } \\
\text { image } \\
\text { moments }\end{array}$ & $\begin{array}{l}\text { Shape } \\
\text { context }\end{array}$ \\
\hline Wrong matching (\%) & 1.9 & 1.1 & 9.7 & 9.5 \\
\hline $\begin{array}{l}\text { No matched contours } \\
(\%)\end{array}$ & 3.1 & 1.8 & 16.4 & 3.5 \\
\hline
\end{tabular}

Tab. 3: The results of the operation of comparison methods with the similarity measure and distance threshold values for environment with one moving object.

\begin{tabular}{|l|l|l|l|l|}
\hline & $\begin{array}{l}\text { Euclidean } \\
\text { distance }\end{array}$ & $\begin{array}{l}\text { Hausdorff } \\
\text { metric }\end{array}$ & $\begin{array}{l}\text { Invariant } \\
\text { image } \\
\text { moments }\end{array}$ & $\begin{array}{l}\text { Shape } \\
\text { context }\end{array}$ \\
\hline Wrong matching (\%) & 0.5 & 0.5 & 2.1 & 1.5 \\
\hline $\begin{array}{l}\text { No matched contours } \\
(\%)\end{array}$ & 2.3 & 1.9 & 23.6 & 10.8 \\
\hline
\end{tabular}

Tab. 4: The results of the operation of comparison methods with the similarity measure and distance threshold values for environment with two moving object.

\begin{tabular}{|l|l|l|l|l|}
\hline & $\begin{array}{l}\text { Euclidean } \\
\text { distance }\end{array}$ & $\begin{array}{l}\text { Hausdorff } \\
\text { metric }\end{array}$ & $\begin{array}{l}\text { Invariant } \\
\text { image } \\
\text { moments }\end{array}$ & $\begin{array}{l}\text { Shape } \\
\text { context }\end{array}$ \\
\hline Wrong matching (\%) & 0.7 & 0.7 & 1.9 & 0.7 \\
\hline $\begin{array}{l}\text { No matched contours } \\
(\%)\end{array}$ & 4.3 & 2.2 & 24.2 & 12.3 \\
\hline
\end{tabular}

\section{Conclusions}

As a result of experiments, the least percent of wrong matched or not matched segments showed by methods based on Hausdorff and Euclid metrics. The proposed methods let determine the correspondence of the segments contours extracted from the data of the laser scanning system. Using the matched segments make it possible to determine the object displacement vector on the scans over time and to make a conclusion about its movement.

\section{References and Notes}

[1] Flusser, J., Tomas S. (2006). Rotation Moment Invariants for Recognition of Symmetric Objects. IEEE Transactions on Image Processing 15.12, pp. 3784-3790.

[2] Andreev, A., Kirov N. (2009). Word Image Matching Based on Hausdorff Distances. Proceedings of the International Conference on Document Analysis and Recognition, ICDAR. N.p., pp. 396-400.

[3] Belongie, S., Jitendra M., Puzicha J. (2002). Shape Matching and Object Recognition Using Shape Contexts. IEEE Transactions on Pattern Analysis and Machine Intelligence 24.4, pp. 509-522.

[4] Memoli, F. (2008). Gromov-Hausdorff Distances in Euclidean Spaces. IEEE Computer Society Conference on Computer Vision and Pattern Recognition Workshops, CVPR Workshops. N.p..

[5] Kazbekov A.V., Maksimov N.A. (2012). Methods for comparing contours in problems of pattern recognition (Metody sravneniya konturov $v$ zadachah raspoznavaniya obrazov). Nauchniy Vestnik MGTU GA., No. 185, pp. 37-43

[6] Skvortsov V.A. (2002). Examples of metric spaces (Primery metricheskih prostranstv). Bibl. Math. Prosveshenie, no. 16.

[7] Shapiro, M.D., Blaschko, M.B. (2004). On Hausdorff Distance Measures, Technical Report UM-CS-2004-071, Departament of Computer Science, University of Massachusetts Amherst.

[8] Hu, Ming-Kuei. (1962). Visual Pattern Recognition by Moment Invariants. Information Theory, IEEE Transactions on 8, pp. 179-187.

\section{Biographical notes}

Andrei Nikitin, is a Ph.D. student in mechatronics and robotics application at Izhevsk State Technical University (ISTU). He was born in 1991 in Izhevsk, Russia. He received the B.Sc. and M.Sc degrees from ISTU in 2013 and 2015 respectively. His research interests are focused on image analysis and technical vision.

Timur Mazitov, is also a Ph.D. student mechatronics and robotics application at ISTU. He was born in 1991 and obtained the B.SC. and M.Sc degrees from ISTU in 2012 and 2014 respectively. His research interests are focused on image and data analysis, mobile robots navigation systems. 